\title{
Pediatric sinonasal rhabdomyosarcoma: A case report
}

\author{
ASLI BOSTANCI, MEHMET ASIK and MURAT TURHAN
}

Department of Otolaryngology, Head and Neck Surgery, Akdeniz University School of Medicine, Antalya 07070, Turkey

Received October 29, 2014; Accepted October 1, 2015

DOI: $10.3892 / \mathrm{etm} .2015 .2839$

\begin{abstract}
Pediatric sinonasal rhabdomyosarcoma (PSR) is a rare condition, with a limited number of previously reported cases. It is an aggressive malignancy associated with poor outcomes, and no consensus has been reached on the optimal therapeutic strategy. The present study reported the case of embryonal PSR in a 2-year old girl, presenting with unilateral nasal obstruction and a polypoid mass protruding from the left nasal cavity. The pediatric patient was initially treated with surgical resection, followed by adjuvant chemotherapy containing vincristine $\left(1.5 \mathrm{mg} / \mathrm{m}^{2}\right.$, weekly) and actinomycin-D $\left(1.5 \mathrm{mg} / \mathrm{m}^{2}\right.$, three times weekly). On the 10 th month of follow-up, tumor recurrence was detected and a salvage surgery was performed, while the same chemotherapy regimen was resumed. Following the first cycle of chemotherapy, the patient developed a fungal bronchopneumonia and succumbed due to disease progression, acute respiratory distress syndrome and septic shock 12 months after diagnosis.
\end{abstract}

\section{Introduction}

Rhabdomyosarcoma is the most common childhood sarcoma. It is a highly aggressive malignant tumor arising in the mesenchymal cells and developing in the striated muscle (1). This particular tumor may appear in any organ or tissue of the body, with the exception of the bone. Approximately $35 \%$ of rhabdomyosarcomas occur in the head and neck region, with $\sim 50 \%$ of them arising in the parameningeal sites, including the paranasal sinuses and nasal cavity (2).

Due to the rarity and largely unknown biological behavior of rhabdomyosarcomas, the management of pediatric sinonasal rhabdomyosarcoma (PSR) remains challenging. The cure rate for localized disease has increased over the past 30 years due to the use of multimodal therapy, which includes surgery and multiagent chemotherapy with or without radiotherapy $(1,3)$. However, the majority of the patients have a poor prognosis, since the tumor has a tendency towards early and extensive local and metastatic dissemination (4).

Correspondence to: Dr Asli Bostanci, Department of Otolaryngology, Head and Neck Surgery, Akdeniz University School of Medicine, 1 Dumlupinar Street, Antalya 07070, Turkey

E-mail: draslibostanci@gmail.com

Key words: rhabdomyosarcoma, pediatric, sinonasal
The present study reported the case of a 2-year old girl with rapid progressive clinical course of PSR, despite multimodal therapy, including primary and salvage surgeries and chemotherapy.

\section{Case report}

The present study was conducted in accordance with the Declaration of Helsinki and was approved by the Ethics Committee of the Akdeniz University School of Medicine (Antalya, Turkey). Written informed consent was obtained from the patient's family.

A 2-year old girl was referred to the Akdeniz University School of Medicine in August 2013 with a 3-month history of unilateral nasal obstruction and purulent rhinorrhea. Multiple medications, including amoxicillin clavulanate, clarithromycin, hypertonic nasal irrigation solutions and nasal decongestants, prescribed by several different specialists due to diagnosis of rhinosinusitis failed to improve the symptoms. Physical examination showed a hemorrhagic, fragile and polypoid tumoral mass within the left nasal cavity. A paranasal computed tomography $(\mathrm{CT})$ scan revealed an irregular mass filling the entire left maxillary sinus, ethmoidal sinus and nasopharynx (Fig. 1). Biopsy samples were obtained for definitive histopathological analysis. Mitotically active, small-round tumor cells with a high nucleocytoplasmic ratio were detected upon microscopic examination (Fig. 2A). Immunohistochemical staining showed that the tumor exhibited strong immunoreactivity for CD56 and desmin (Fig. 2B-C); however, no staining was observed for myogenin, CD99 and CD20. These histological and immunohistochemical findings were consistent with a diagnosis of embryonal rhabdomyosarcoma.

The tumor was completely resected using an endoscopic approach and the surgical margins were found to be negative for tumor infiltration, while metastatic work-up was negative for systemic dissemination. Thus, the patient was diagnosed with stage IB disease, according to the Intergroup Rhabdomyosarcoma Study (IRS) staging system (5). A total of six cycles of adjuvant chemotherapy with vincristine $\left(1.5 \mathrm{mg} / \mathrm{m}^{2}\right.$, weekly) and actinomycin-D $\left(1.5 \mathrm{mg} / \mathrm{m}^{2}\right.$, three times weekly) were administered with no severe adverse events. On the 10th month of follow-up (June 2014), a paranasal CT scan revealed a local-regional recurrence within the left nasal cavity. Salvage surgery was performed in order to remove the recurrent tumor using an endoscopic approach. The recurrent tumor was completely resected and histopathological analysis indicated a diagnosis of recurrent rhabdomyosarcoma. The 


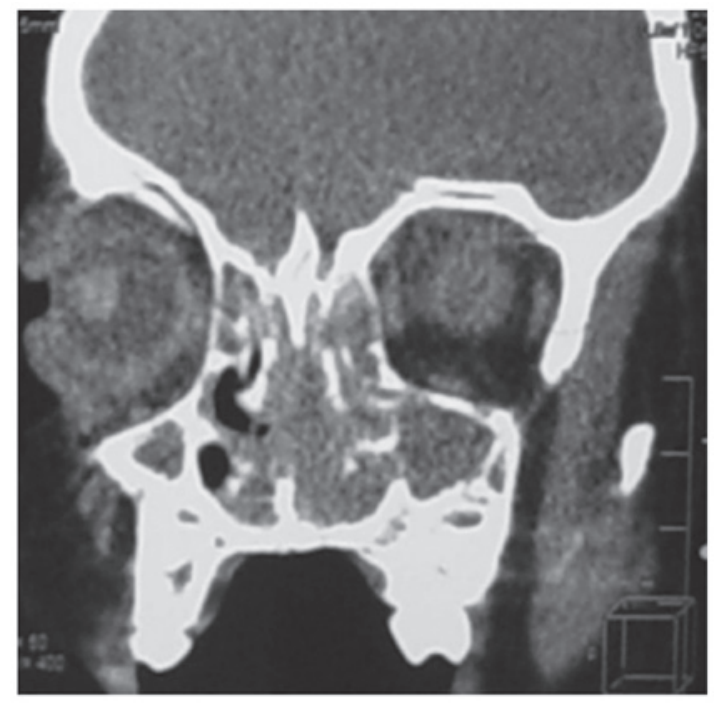

Figure 1. Paranasal computed tomography scan of rhabdomyosarcoma filling the entire left maxillary sinus, ethmoidal cells and nasopharynx.

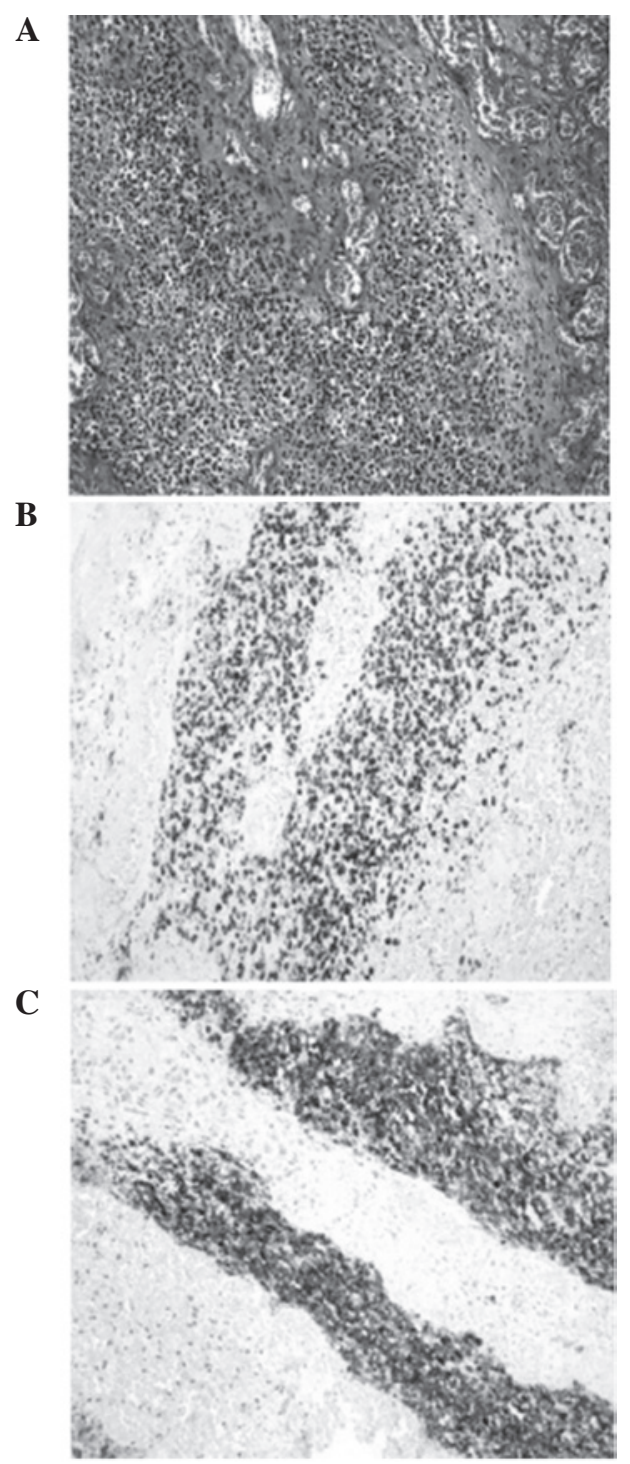

Figure 2. Embryonal rhabdomyosarcoma. Mitotically active, small-round tumor cells with a high nucleocytoplasmic ratio were observed, based on staining using (A) hematoxylin and eosin (magnification, x10), (B) desmin (magnification, x20) and (C) CD56 (magnification, x20). same chemotherapy regimen was resumed, following salvage surgery. The pediatric patient developed a fungal bronchopneumonia subsequent to the first cycle of chemotherapy and was further treated with intravenous antifungal antibiotics. However, medical treatment failed to improve the clinical condition of the patient, and she was admitted to the Intensive Care Unit. The results of blood gas test and chest radiography, which revealed diffuse pulmonary infiltrates, were compatible with acute respiratory distress syndrome (ARDS). The patient succumbed due to disease progression, ARDS and septic shock 12 months after diagnosis (August 2014).

\section{Discussion}

PSR, particularly at its earliest stages, produces symptoms that may be incorrectly attributed to allergy and infection. Nasal obstruction, epistaxis and drainage are often exhibited prior to the presentation of more severe symptoms, such as cranial neuropathy (4). As observed in the present case, these indistinct symptoms may impede the early diagnosis and treatment of the disease. All patients suffering from these symptoms should therefore undergo an initial complete clinical evaluation of their medical history, physical examination and nasopharyngoscopy. Endoscopic evaluation usually reveals a unilateral, polypoid mass (6), and a biopsy should be performed to rule out the possibility of an invasive tumor.

Although pediatric rhabdomyosarcoma is a morphologically and genetically heterogeneous tumor, two cell types are common, the embryonal and alveolar types, each characterized by specific genetic alterations (7). The embryonal type is the most common histological type of head and neck rhabdomyosarcoma (2), and has been linked to the loss of homozygosity on chromosome 11p15, the location of insulin-like growth factor II. By contrast, alveolar tumors often have a chromosomal translocation $[\mathrm{t}(2 ; 13)(\mathrm{q} 35 ; \mathrm{q} 14)]$ that fuses the $P A X 3$ gene with the FKHR gene, contributing to tumorigenesis (7). In contrast to other mucosa-associated head and neck rhabdomyosarcomas, PSR predominantly shows an alveolar histology, particularly the solid alveolar type (8). Using immunohistochemical staining, PSR shows positive immunoreactivity for desmin and myogenin, and negative immunoreactivity for cytokeratin, epithelial membrane antigen, CD45, CD99 and S-100 protein (9). The majority of alveolar tumors are strongly positive for myogenin, whereas embryonal tumors often exhibit positivity to a lesser degree $(9,10)$, which is in agreement with the immunohistochemistry results of the present case.

The embryonal and alveolar subtypes of pediatric rhabdomyosarcoma have diverse prognostic factors and outcomes, with 5-year failure-free survival rates ranging between 31 and $87 \%$ according to the patient age, stage, histological type and tumor site (11). The overall outcomes of patients with the alveolar histology are worse than those of patients with the embryonal histology. In addition, due to its parameningeal location, PSR has a potential for cerebrospinal fluid dissemination, which contributes to a poor prognosis (12). Thereby, early diagnosis and optimal management are important in reducing morbidity and mortality. Comprehensive imaging studies, including paranasal CT or magnetic resonance imaging scans, should be performed in order to anatomically localize the tumor site and extent of the disease, and to determine the correct disease stage. 
The treatment of pediatric rhabdomyosarcoma often requires a multimodal therapy, including surgery, chemotherapy and radiotherapy (3); however, it is not currently possible to determine the best treatment strategy on the basis of high-level scientific evidence, since previous IRS trials comprise a heterogeneous population and do not evaluate the treatment outcomes specifically for PSR (11). The surgical goal is the complete excision of the primary tumor, whenever possible, without causing major functional or cosmetic deficits. Incompletely resected tumors or tumors with a positive resection margin are generally treated with radiotherapy (13). Novel techniques of implementing radiotherapy, including intensity-modulated, proton beam radiotherapy and brachytherapy may reduce long-term sequelae, as compared with three-dimensional conformal radiotherapy (14). In addition, all patients with rhabdomyosarcoma require treatment with chemotherapy (15). The most commonly used regimens include vincristine and actinomycin-D in combination with an alkylating agent, such as cyclophosphamide or ifosfamide. Compared with tumors in other regions, PSRs have a decreased response to treatment, a relapse rate of 30-40\% and a greater potential for early metastasis (16). The majority of rhabdomyosarcoma recurrences involve the primary site or adjacent region. Although salvage surgery is advocated for resectable residual or recurrent PSR, the evidence for its efficacy is considerably limited $(15,16)$.

In conclusion, PSR is a rare aggressive malignancy associated with poor outcomes. Only a limited number of PSR cases have previously been reported $(2,4,6,8,11,13,14)$. The present report emphasized the prognostic significance of the tumor site and the age upon diagnosis of rhabdomyosarcoma, as well as the necessity of a multidisciplinary management strategy in clinical centers with adequate experience.

\section{References}

1. McDowell HP: Update on childhood rhabdomyosarcoma. Arch Dis Child 88: 354-357, 2003.
2. Hicks J and Flaitz C: Rhabdomyosarcoma of the head and neck in children. Oral Oncol 38: 450-459, 2002.

3. Walterhouse D and Watson A: Optimal management strategies for rhabdomyosarcoma in children. Paediatr Drugs 9: 391-400, 2007.

4. Herrmann BW, Sotelo-Avila C and Eisenbeis JF: Pediatric sinonasal rhabdomyosarcoma: Three cases and a review of the literature. Am J Otolaryngol 24: 174-180, 2003.

5. Lawrence W Jr, Anderson JR, Gehan EA and Maurer H: Pretreatment TNM staging of childhood rhabdomyosarcoma: A report of the Intergroup Rhabdomyosarcoma Study Group. Children's Cancer Study Group. Pediatric Oncology Group.Cancer 80: 1165-1170, 1997.

6. Fyrmpas G, Wurm J, Athanassiadou F, Papageorgiou T, Beck JD, Iro $\mathrm{H}$ and Constantinidis J: Management of paediatric sinonasal rhabdomyosarcoma. J Laryngol Otol 123: 990-996, 2009.

7. Pappo AS, Shapiro DN, Crist WM and Maurer HM: Biology and therapy of pediatric rhabdomyosarcoma. J Clin Oncol 13: 2123-2139, 1995.

8. Ahmed AA and Tsokos M: Sinonasal rhabdomyosarcoma in children and young adults. Int J Surg Pathol 15: 160-165, 2007.

9. Kumar S, Perlman E, Harris CA, Raffeld M and Tsokos M: Myogenin is a specific marker for rhabdomyosarcoma: An immunohistochemical study in paraffin-embedded tissues. Mod Pathol 13: 988-993, 2000.

10. Cessna MH, Zhou H, Perkins SL, Tripp SR, Layfield L, Daines C and Coffin CM: Are myogenin and MyoD1 expression specific for rhabdomyosarcoma? A study of 150 cases, with emphasis on spindle cell mimics. Am J Surg Pathol 25: 1150-1157, 2001.

11. Meza JL, Anderson J, Pappo AS and Meyer WH; Children's Oncology Group: Analysis of prognostic factors in patients with nonmetastatic rhabdomyosarcoma treated on intergroup rhabdomyosarcoma studies III and IV: The Children's Oncology Group. J Clin Oncol 24: 3844-3851, 2006.

12. Sturgis EM and Potter BO: Sarcomas of the head and neck region. Curr Opin Oncol 15: 239-252, 2003.

13. Daya H, Chan HS, Sirkin W and Forte V: Pediatric rhabdomyosarcoma of the head and neck: Is there a place for surgical management? Arch Otolaryngol Head Neck Surg 126: 468-472, 2000 .

14. Gosiengfiao Y, Reichek J and Walterhouse D: What is new in rhabdomyosarcoma management in children? Paediatr Drugs 14: 389-400, 2012

15. Buwalda J, Blank LE, Schouwenburg PF, Copper MP, Strackee SD, Voûte PA, Merks JH and Caron HN: The AMORE protocol as salvage treatment for non-orbital head and neck rhabdomyosarcoma in children. Eur J Surg Oncol 30: 884-892, 2004

16. Paulino AC, Bauman N, Simon JH, Nguyen TX, Ritchie JM and Tannous R: Local control of parameningeal rhabdomyosarcoma: Outcomes in non-complete responders to chemoradiation. Med Pediatr Oncol 41: 118-122, 2003. 